\title{
Nab-paclitaxel plus tegafur gimeracil oteracil potassium capsule (S-1) as first-line treatment for advanced biliary tract adenocarcinoma: a phase 2 clinical trial
}

\author{
Wen Zhang", Yongkun Sun", Zhichao Jiang, Wang Qu, Caifeng Gong, Aiping Zhou \\ Department of Medical Oncology, National Cancer Center/National Clinical Research Center for Cancer/Cancer Hospital, Chinese Academy of \\ Medical Sciences and Peking Union Medical College, Beijing, China \\ Contributions: (I) Conception and design: A Zhou, Y Sun; (II) Administrative support: None; (III) Provision of study materials or patients: None; (IV) \\ Collection and assembly of data: Y Sun, W Zhang; (V) Data analysis and interpretation: Y Sun, W Zhang; (VI) Manuscript writing: All authors; (VII) \\ Final approval of manuscript: All authors. \\ \#These authors contributed equally to this work. \\ Correspondence to: Dr. Aiping Zhou. Department of Medical Oncology, National Cancer Center/National Clinical Research Center for Cancer/ \\ Cancer Hospital, Chinese Academy of Medical Sciences and Peking Union Medical College, Beijing 100021, China. Email: aiping_zhou@yeah.net.
}

\begin{abstract}
Background: This study aimed to evaluate the efficacy and safety of a new combination of nab-paclitaxel plus tegafur gimeracil oteracil potassium capsule (S-1) for patients with advanced biliary tract carcinoma (BTC).

Methods: Patients were treated with nab-paclitaxel at a dose of $125 \mathrm{mg} / \mathrm{m}^{2}$ on day 1 and 8 , and S-1, 80 to $120 \mathrm{mg} /$ day on days $1-14$ of a 21-day cycle. Treatments were repeated until disease progression or unacceptable toxicity occurred. The primary endpoint was objective response rate (ORR). The secondary endpoints were median progression-free survival (PFS), overall survival (OS), and adverse events (AEs).

Results: The number of patients enrolled were 54, and 51 patients were evaluated for efficacy. A total of 14 patients achieved partial response (PR) with an ORR of $27.5 \%$. The ORR varied by sites, with 53.8\% (7/13) for gallbladder carcinoma, 18.4\% (7/38) for cholangiocarcinoma. The most common grade 3 or 4 toxicities were neutropenia and stomatitis. The median PFS and OS were 6.0 and 13.2 months, respectively.

Conclusions: The combination of nab-paclitaxel with S-1 showed explicit antitumor activities and favorable safety profile in advanced BTC and could serve as a potential non-platinum and -gemcitabinebased regimen.
\end{abstract}

Keywords: Biliary tract adenocarcinoma; chemotherapy; nab-paclitaxel; tegafur gimeracil oteracil potassium capsule (S-1)

Submitted Apr 30, 2021. Accepted for publication Sep 10, 2021. Published online Oct 18, 2021.

doi: $10.21037 / \mathrm{hbsn}-21-172$

View this article at: https://dx.doi.org/10.21037/hbsn-21-172

\section{Introduction}

Biliary tract carcinoma (BTC) is a group of rare malignant diseases originating from the hepatobiliary tract, which is further categorized into 3 entities based on the originating site: intrahepatic cholangiocarcinoma (iCCA), extrahepatic cholangiocarcinoma (eCCA), and gallbladder cancer (GBC). BTC represents $3 \%$ of all gastrointestinal neoplasms in adults (1). However, the incidence of BTC is increasing worldwide, mainly due to iCCA (2). The 3 sub-entities are heterogeneous in genetic and clinical presentation. iCCA is charactered by the fibroblast growth factor receptor-2 (FGFR2) fusion (14-23\%) (3-5) and isocitrate dehydrogenase (IDH) mutation (10-20\%) (6), whereas GBC by HER-2 amplification (5-15\%) (7). Generally, the 
prognosis of BTC is extremely poor due to its insidious onset, advanced stage at diagnosis, and limited effective treatment options for advanced disease. The overall 5-year survival rate of $\mathrm{BTC}<5 \%$ (8).

Although advances in precision targeted therapy have been applied to advanced BTC, systemic chemotherapy remains the standard care in first-line treatment. In most recent years, novel agents targeting aberrant FGFR and $I D H 1$ genes have shown promising activities against advanced BTC in the second- or later-line treatment and were approved by the Food and Drug Administration (US FDA) for such indication $(9,10)$. However, these agents can be only applied to a small portion of the patients with BTC. Checkpoint inhibitors alone are reported to have the modest activities with an objective response rate (ORR) varied from $5 \%$ to $20 \%$ and the combination strategy is now under broad investigation with inconsistent results reported from some phase I or II trials (11). Although limited, gemcitabine plus cisplatin (GC), the most widely used chemotherapy regimen for patients with advanced BTC, yields consistent and definite efficacy with an ORR around $26 \%$ and an overall survival (OS) of 11.7 months (12). Gemcitabine plus oxaliplatin has shown similar activities with GC in phase II trials and are recommended as alternative regimen by National Comprehensive Cancer Network (NCCN) guideline (13). Although chemotherapy will stand as the major strategy for the first-line treatment for most advanced BTC, there is still a bigger need to develop novel chemotherapy regimens.

S-1 is a fluorouracil derivative that showed good singledrug antitumor activity in pancreatic, biliary, gastric cancer, and other digestive tract tumors. The results of a Japanese phase III randomized trial (FUGA-BT) demonstrated that the combination of gemcitabine plus S-1 (GS) was noninferior to the standard of care of GC with a median OS of 15.1 and 13.4 months, respectively, and a good tolerability profile (14).

Nab-paclitaxel is a novel taxane with the advantage of less vehicle-related hypersensitivity reactions, neurotoxicity, and neutropenia, with wide antitumor spectrum. The regimen of nab-paclitaxel and gemcitabine has been approved for advanced pancreatic cancer (15). This combination also showed activities in advanced cholangiocarcinoma in a phase 2 clinical trial, in which 74 patients with advanced cholangiocarcinoma were enrolled (16). Although the study failed to meet its primary end point of improvement in progression-free survival (PFS) rate at 6 months (the actual 6-month PFS rate of $61 \%$ was insufficient to achieve the expected hypothesis of 70\%); however, an ORR of $30 \%$, median OS of 12.4 months, suggesting that nabpaclitaxel may be a new candidate partner for combined chemotherapy.

Nab-paclitaxel and S-1 have synergistic antitumor effect in a preclinical model (17). This combination regimen achieved a favorable ORR in advanced pancreatic cancer and was well tolerated $(18,19)$. Thus, we conducted a phase 2 trial to evaluate the efficacy and safety of nab-paclitaxel with $\mathrm{S}-1$ as the first-line treatment for advanced biliary tract adenocarcinoma. Here, we reported the final results. We present the following article in accordance with the TREND reporting checklist (available at https://hbsn. amegroups.com/article/view/10.21037/hbsn-21-172/rc).

\section{Methods}

\section{Study design}

This was a prospective, single-arm, phase 2 clinical trial. The trial was conducted at the Cancer Hospital \& Institute, Chinese Academy of Medical Sciences. The trial was approved by the institutional ethics committee. The trial was conducted in accordance with Declaration of Helsinki (as revised in 2013) and the Good Clinical Practice Guidelines. All patients provided written informed consent before enrollment. The registration number in ClinicalTrials.gov was NCT03830606.

\section{Eligibility criteria}

The main eligibility criteria included: age over 18 years, pathologically confirmed biliary tract adenocarcinoma, unresectable, recurrent or metastatic lesions, Eastern Cooperative Oncology Group (ECOG) performance status of 0 or 1 , no history of chemotherapy or an interval of $>6$ months since the last dose of adjuvant chemotherapy, adequate function of major organs, neutrophil count $\geq 1.5 \times 10^{9} / \mathrm{L}$, number of platelets $\geq 100 \times 10^{9} / \mathrm{L}$, hemoglobin level $\geq 9.0 \mathrm{~g} / \mathrm{dL}$, aspartate aminotransferase and alanine aminotransferase $\leq 1.5 \times$ upper limit of reference standard ( $\leq 5 \times$ upper limit of reference standard for patient with liver metastases), total bilirubin $\leq 1.5 \mathrm{mg} / \mathrm{dL}$, serum creatinine $\leq 1.5 \mathrm{mg} / \mathrm{dL}$.

\section{Treatment}

Patients received nab-paclitaxel $\left(125 \mathrm{mg} / \mathrm{m}^{2}\right)$ infusion 
Table 1 Patient characteristics

\begin{tabular}{|c|c|c|c|c|}
\hline Characteristic & Total $(n=54)$ & GBC $(n=13)$ & iCCA $(n=35)$ & $\operatorname{eCCA}(n=6)$ \\
\hline Age, median [range] & 58 [34-73] & 59 [48-67] & 58 [34-73] & 62 [60-67] \\
\hline Prior surgery, n (\%) & $18(33.3)$ & $7(53.8)$ & $6(17.1)$ & 5 (83.3) \\
\hline \multicolumn{5}{|l|}{ cTNM staging, n (\%) } \\
\hline IV & $52(96.3)$ & $13(100.0)$ & $33(94.3)$ & $6(100.0)$ \\
\hline \multicolumn{5}{|l|}{ ECOG score, n (\%) } \\
\hline 0 & $41(75.9)$ & $9(69.2)$ & $27(77.1)$ & $5(83.3)$ \\
\hline 1 & $13(24.1)$ & $4(30.8)$ & 8 (22.9) & $1(16.7)$ \\
\hline
\end{tabular}

GBC, gallbladder cancer; iCCA, intrahepatic cholangiocarcinoma; eCCA, extrahepatic cholangiocarcinoma; cTNM, Tumor, Nodes, Metastasis classification of staging; ECOG, Eastern Cooperative Oncology Group; CA19-9, carbohydrate antigen 19-9.

on day 1 and 8 . S-1 was administered orally twice daily $\left[80 \mathrm{mg} /\right.$ day for a body surface area $\left(\right.$ BSA) $<1.25 \mathrm{~m}^{2}$, $100 \mathrm{mg} /$ day for a BSA between 1.25 and $1.5 \mathrm{~m}^{2}$, $120 \mathrm{mg} /$ day for a BSA $>1.5 \mathrm{~m}^{2}$ ] on day 1 to 14 , repeated every 3 weeks. Patients with partial response (PR) or stable disease (SD) were recommended to receive $\mathrm{S}-1$ as maintenance treatment after a maximum of 6 cycles of the combination therapy. Treatment was permitted until disease progression or development of an unacceptable toxic effect or withdraw from informed consent.

\section{Assessments and endpoints}

The primary endpoint was ORR. Secondary endpoints included PFS, OS, and safety. Tumor response was evaluated every 2 cycles according to the Response Evaluation Criteria in Solid Tumors 1.1 criteria (RECIST 1.1). Toxicities were measured by the National Cancer Institute-Common Terminology Criteria for Adverse events, version 4.0 (NCI-CTCAE 4.0). PFS was calculated from study entry to disease progression or death whichever occurred first. OS was calculated from study entry to death for any cause.

\section{Statistical analysis}

According to the ABC-02 study, chemotherapy with GC led to an ORR of $26.1 \%$. We hypothesized that chemotherapy with nab-paclitaxel plus S1 would further improve the ORR from $26 \%$ to $40 \%$. A sample size was 49 with a one-side type I error of 0.1 and a power of $80 \%$, and with $10 \%$ of dropout rate considered, the intended number of patients was 54. All statistical analyses were conducted with SPSS 22.0 software.

\section{Results}

\section{Patient characteristics}

Between March 2016 and September 2018, 54 eligible patients were enrolled. The baseline demographic and clinical characteristics are shown in Table 1 . The median age was 58 (range, 34-73) years, including 27 women (50.0\%). Among the 54 patients, 13 (24.1\%) were GBC, 35 (64.8\%) of iCCA, and $6(11.1 \%)$ of eCCA.

\section{Administered treatment and efficacy}

Patient disposition is summarized in Figure 1. The median number of cycles was 4 (range, 1-6). A total of 12 patients subsequently received maintenance therapy with $\mathrm{S}-1$ for a median of 5 (range, 2-8) cycles. Two patients with iCCA and 1 patient with GBC had remarkable tumor shrinkage after chemotherapy and underwent hepatobiliary surgery resection.

Among the 54 enrolled patients, 1 patient died of gastrointestinal bleeding just after the first dose of nab- 


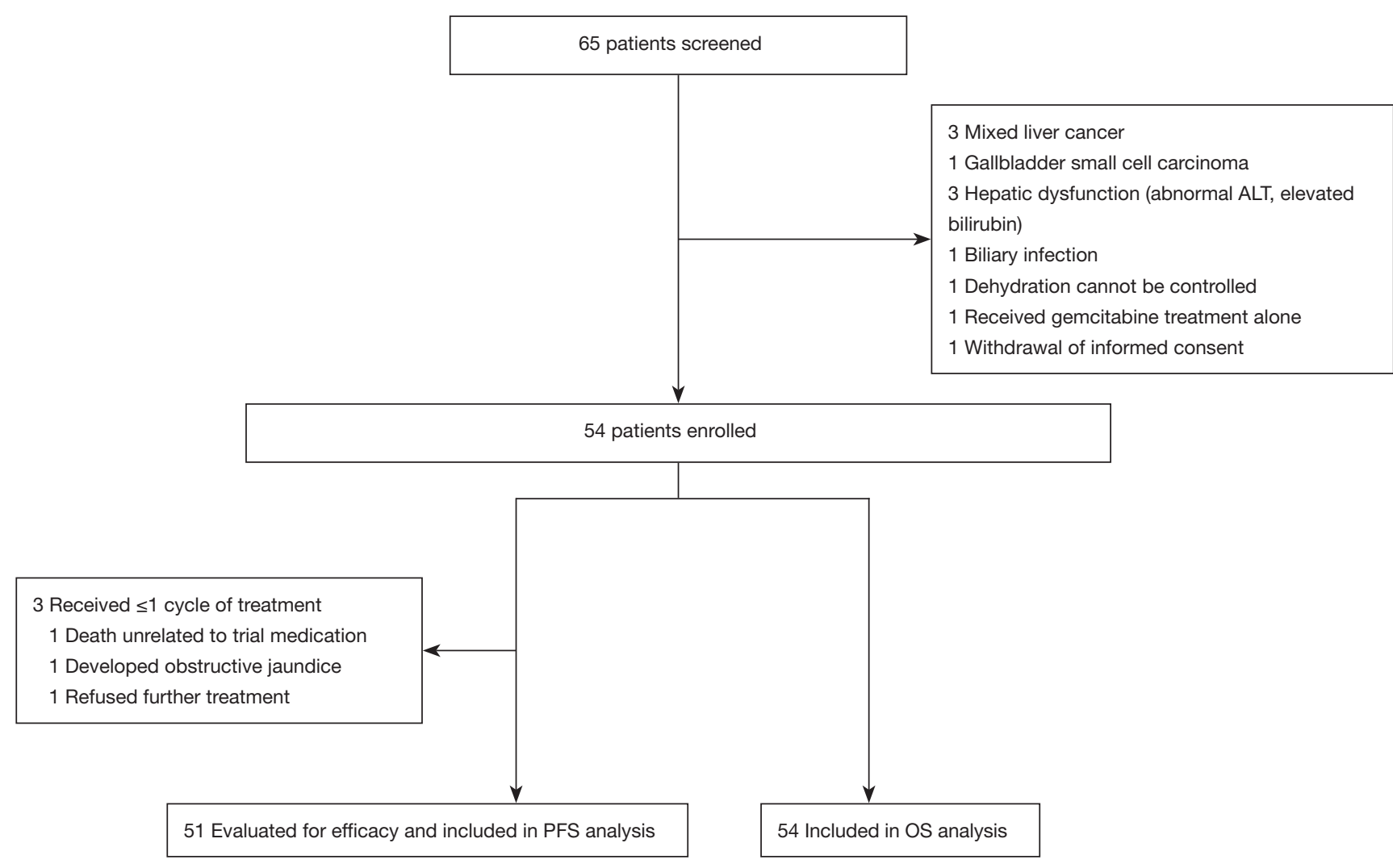

Figure 1 Flowchart summarizing patients' disposition. ALT, alanine aminotransferase; PFS, progression-free survival; OS, overall survival.

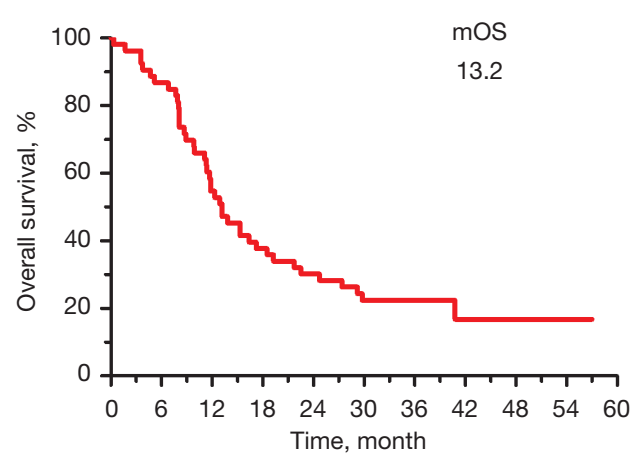

Figure 2 OS in the whole group. mOS, median overall survival.

paclitaxel, 1 patient refused further treatment, and 1 patient developed obstructive jaundice during the first cycle. Except the above 3 patients, the rest 51 patients were evaluable for efficacy. In all, 14 (27.5\%) patients achieved PR, 22 (43.1\%) $\mathrm{SD}$, and 15 (29.4\%) progression disease (PD), yielding the ORR of $27.5 \%$. The disease control rate (DCR) was $70.6 \%$.
The response rates varied by primary tumor site: $53.8 \%$ [7/13] in GBC, $18.4 \%$ [7/38] for cholangiocarcinoma \{18.2\% [6/33] for iCCA, and 20.0\% [1/5] for eCCA\}.

After failure of the first-line therapy, 24/51 (47.1\%) patients received subsequent therapy. Among the 24 patients, $15(62.5 \%)$ patients received a chemotherapy regimen of gemcitabine plus a platinum, $13(54.2 \%)$ patients received a VEGF-TKI (including anlotinib, lenvatinib, apatinib, and regorafenib), and 8 (33.3\%) patients received immune checkpoint inhibitors (including PD-1 antibody and PD-L1 antibody).

As of January 1, 2021, with a median follow-up of 39 (range, 27-58) months, the median OS was 13.2 (95\% CI: 10.3-16.0) months (Figure 2), and the median PFS was 6.0 (95\% CI: 4.2-7.7) months (Figure 3). The median overall survival (mOS) were 9.9 (95\% CI: 3.7-16.0) and 13.8 (95\% CI: 10.0-17.5) months, median progression-free survival (mPFS) were 4.5 (95\% CI: 2.1-6.8) and 6.0 (95\% CI: 3.9-8.0) months for GBC and cholangiocarcinoma patients, respectively (Figures 4,5 ). 


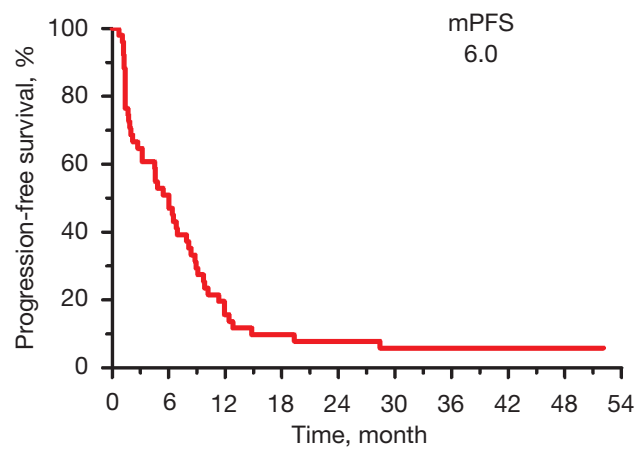

Figure $3 \mathrm{PFS}$ in the whole group. mPFS, median progression-free survival.

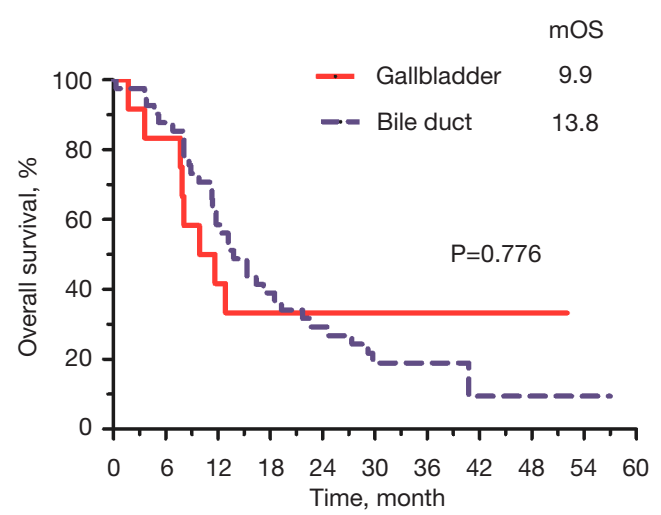

Figure 4 OS curves by primary sites. mOS, median overall survival.

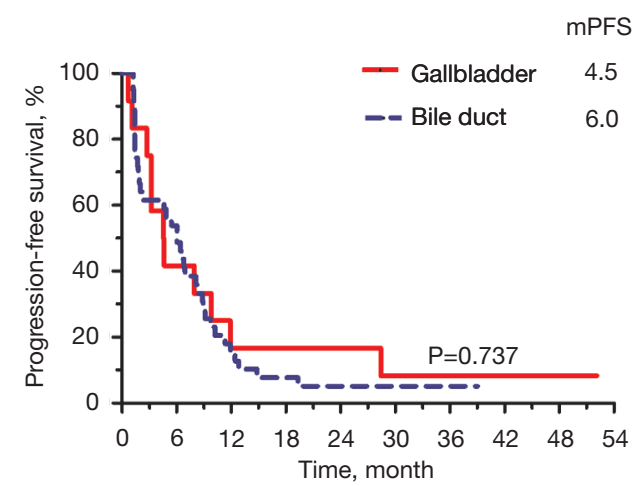

Figure 5 PFS curves by primary sites. mPFS, median progressionfree survival.

\section{Safety}

The most common treatment-related adverse events were hair loss [33 patients $(61.1 \%)$ ], neutropenia [31 patients $(57.4 \%)$ ], peripheral neuropathy [23 patients $(42.6 \%)$ ], anemia [19 patients $(35.2 \%)]$, and stomatitis [17 patients (31.5\%)] (Table 2). Overall, 35 patients (64.8\%) experienced a grade 3 or higher treatment-related adverse event, mainly including neutropenia in 17 (31.5\%) patients, stomatitis in $4(7.4 \%)$ patients. There were no treatment-related deaths.

\section{Discussion}

In this study, the combination of nab-paclitaxel and S-1 achieved an ORR of $27.5 \%$ in 51 evaluable patients with advanced BTC. The ORR failed to meet the expected endpoint level of $40 \%$. However, the results suggested that nab-paclitaxel and S-1 seemed to have comparable activity to that of the standard gemcitabine-based doublet chemotherapy. The ORR of GC regimen was $26.1 \%$ in the early ABC-02 study (12) and $32.4 \%$ in the more recent Japanese JCOG1113 study (14). The ORR of GEMOX, another world widely used gemcitabine-based regimen, was $23 \%$ in the BINGO study (20). In the JCOG1113 study, GS was shown non-inferior to GC, with an ORR of $29.4 \%$. Even the most recently reported doublet of nab-paclitaxel and gemcitabine only achieved an ORR of $30 \%$ by Sahai (16).

Moreover, the combination of nab-paclitaxel and S-1 exhibited an encouraging PFS of 6.0 months and OS of 13.2 months in this study. This is also comparable to that of the gemcitabine-base regimen. In JCOG1113 study, PFS of 5.8 and 6.8 months, OS of 13.4 and 15.1 months were reported with GC and GS, respectively. The OS of nabpaclitaxel plus gemcitabine was 13.0 months (16). However, the OS was only 11.7 months with GC in the early ABC02 study. All the above data reflect the fact that there is still an unmet need to develop more efficient chemotherapy regimen.

A strategy of triplet chemotherapy has been investigated in some clinical trials. In a phase III trial reported by Sakai (21), gemcitabine-cisplatin, and S-1 (GCS) was proven to have a significant improvement in ORR from $15 \%$ to 
Table 2 Adverse events

\begin{tabular}{lcc}
\hline \multirow{2}{*}{ Adverse events } & \multicolumn{2}{c}{ Incidence, $\mathrm{n}(\%)$} \\
\cline { 2 - 3 } Neutropenia & Grade 1/2 & Grade 3/4 \\
Anemia & $19(35.2)$ & $17(31.5)$ \\
Thrombocytopenia & $11(20.4)$ & $0(0.0)$ \\
ALT elevation & $4(7.4)$ & $1(1.9)$ \\
TBIL elevation & $10(18.5)$ & $1(1.9)$ \\
Anorexia & $13(24.1)$ & $3(5.6)$ \\
Nausea & $11(20.4)$ & $0(0.0)$ \\
Vomiting & $7(13.0)$ & $0(0.0)$ \\
Stomatitis & $17(31.5)$ & $1(1.9)$ \\
Diarrhea & $10(18.5)$ & $4(7.4)$ \\
asthenia & $8(14.8)$ & $2(3.7)$ \\
Rash & $4(7.4)$ & $2(3.7)$ \\
Hand-foot syndrome & $7(13.0)$ & $0(0.0)$ \\
Peripheral neuropathy & $23(42.6)$ & $1(1.9)$ \\
Hair loss & $33(61.1)$ & $3(5.6)$ \\
\hline ALT, alanine & $0(0.0)$ \\
\hline
\end{tabular}

ALT, alanine transaminase; TBIL, total bilirubin.

41.5\% and PFS from 5.5 to 7.4 months over GC; however, the prolongation of OS was only marginal significant (13.5 months for GCS vs. 12.6 months for GC, $\mathrm{P}=0.046$ ). Shroff et al. reported that the triplet combination of gemcitabine, cisplatin, and nab-paclitaxel produced an ORR of $45 \%$, OS of 19.2 months, in a phase 2 clinical trial (22).

Although randomized phase 3 controlled trials are in progress (23), some single-arm studies with small samples have shown that immune checkpoint inhibitors combined with standard chemotherapy may improve the response rate and OS of advanced BTC. Ueno et al. reported a 37\% ORR in a chemotherapy-naïve Japanese cohort that received nivolumab plus gemcitabine and cisplatin with a median OS of 15.4 months (24). In another single-arm study, 32 patients with advanced BTC were treated with nivolumab plus GC, and the ORR was as high as $55.6 \%$ (25). Nabpaclitaxel and PD-1/PD-L1 inhibitors-based regimen has been explored in a variety of tumors, such as breast cancer and non-small cell lung cancer and showed promising activities and favorable safety $(26,27)$. Due to the favorable safety as discussed below and explicit antitumor activities, it seems interesting to investigate the combination of nab- paclitaxel and S-1 with PD-1/PD-L1 inhibitors in BTC in future.

Interestingly, an ORR of $53.8 \%$ [7/13] was observed in the gall bladder carcinoma subgroup which seemed numerically much higher than that of the $18.4 \%$ (7/38) in the cholangiocarcinoma subgroup in this study. This result gives a hint that gallbladder carcinoma and cholangiocarcinoma might respond variously to chemotherapy. The heterogonous characteristic of the biliary tract cancer provides some backup to the postulation. In ABC-02 study, the ORR of gallbladder carcinoma and cholangiocarcinoma were $37.7 \%$ and $19 \%$, respectively (12). However, this should be interpreted with caution due to the small number of the patients with gall bladder carcinoma. Furthermore, the advantage of ORR seemed not transferred to survival benefit in this study. Nevertheless, the role of nab-paclitaxel and S-1 in gall bladder carcinoma necessitates further investigation.

With respect to adverse reactions, no unexpected AEs were observed in the present study, and the incidence of hematological toxicity was similar to that observed in prior doublet chemotherapy studies (12-14). The incidence of gastrointestinal reactions in the present study seemed a little lower than that of platinum and gemcitabine. For instance, grade 3 or 4 gastrointestinal reactions were observed in $5 \%$ of the patients in this study compared with the previously observed $10 \%$ with gemcitabine plus cisplatin (12). However, neurotic toxicity of nab-paclitaxel increased with the number of treatment cycles. The incidence of grade 3 neurotic toxicity in the present study was $5.5 \%$, which was similar to the $8 \%$ reported by a previous phase 2 study of gemcitabine and nab-paclitaxel (16). With the favorable safety profile, nab-paclitaxel plus S-1 provides a potential new option in case that gemcitabine-cisplatin chemotherapy is not applicable and might act as a favorable candidate partner for checkpoint inhibitors.

\section{Limitations}

The present trial was limited in that it was not a randomized, controlled trial, and included a relatively low number of patients. As a result of these limitations, our findings must be interpreted with caution. We acknowledge that we included a higher percentage of patients with iCCA [64.8\%, compared with $22 \%$ in early study of ABC-01 (28)]. This latter observation is reflective of the changing trends in biliary cancer epidemiology and was also seen in the recent study of gemcitabine, cisplatin 
and nab-paclitaxel by Shroff et al. (63\% of patients with iCCA) (22). A recent retrospective analysis suggests that different subsets of BTCs may be associated with different survival between iCCA, eCCA and GBC (29). It is suggested that the future research should focus on different parts of BTCs. In addition, randomized controlled trials would be carried out to reduce the bias caused by baseline imbalance.

In conclusion, the combination of nab-paclitaxel with S-1 showed explicit activities in advanced biliary carcinoma that seemed comparable to the current standard regimen in terms of ORR, PFS and OS. The safety profile of nabpaclitaxel and S-1 was favorable. This novel combination thus could be a potential non-platinum and -gemcitabinebased regimen for this disease. The higher ORR observed in gallbladder carcinoma subgroup appears interesting and warranted further investigation. In view of other findings, there is a synergistic effect between nab-paclitaxel and PD-1 inhibitor, and we observed that the combination of the two drugs showed excellent preliminary results in another ongoing second-line study of gastric cancer (NCT04140318), we are considering to conduct a phase II study of AS regimen combined with PD-1 inhibitor in the treatment of GBC.

\section{Acknowledgments}

A part of the study results was accepted as an abstract and poster at the American Society of Clinical Oncology (ASCO) 2019 annual meeting I, Chicago, United States, 31 May to 4 June 2019.

Funding: None.

\section{Footnote}

Reporting Checklist: The authors have completed the TREND reporting checklist. Available at https://hbsn. amegroups.com/article/view/10.21037/hbsn-21-172/rc

Data Sharing Statement: Available at https://hbsn. amegroups.com/article/view/10.21037/hbsn-21-172/dss

Conflicts of Interest: All authors have completed the ICMJE uniform disclosure form (available at https://hbsn. amegroups.com/article/view/10.21037/hbsn-21-172/coif). The authors have no conflicts of interest to declare.

Ethical Statement: The authors are accountable for all aspects of the work in ensuring that questions related to the accuracy or integrity of any part of the work are appropriately investigated and resolved. The trial was conducted in accordance with Declaration of Helsinki (as revised in 2013) and the Good Clinical Practice Guidelines. This trial was approved by the institutional ethics committee. All patients provided written informed consent before enrollment. The registration number in ClinicalTrials.gov was NCT03830606.

Open Access Statement: This is an Open Access article distributed in accordance with the Creative Commons Attribution-NonCommercial-NoDerivs 4.0 International License (CC BY-NC-ND 4.0), which permits the noncommercial replication and distribution of the article with the strict proviso that no changes or edits are made and the original work is properly cited (including links to both the formal publication through the relevant DOI and the license). See: https://creativecommons.org/licenses/by-nc-nd/4.0/.

\section{References}

1. Ebata T, Ercolani G, Alvaro D, et al. Current Status on Cholangiocarcinoma and Gallbladder Cancer. Liver Cancer 2016;6:59-65.

2. Bertuccio P, Malvezzi M, Carioli G, et al. Global trends in mortality from intrahepatic and extrahepatic cholangiocarcinoma. J Hepatol 2019;71:104-14.

3. Lowery MA, Ptashkin R, Jordan E, et al. Comprehensive Molecular Profiling of Intrahepatic and Extrahepatic Cholangiocarcinomas: Potential Targets for Intervention. Clin Cancer Res 2018;24:4154-61.

4. Walter D, Hartmann S, Waidmann O. Update on cholangiocarcinoma: potential impact of genomic studies on clinical management. Z Gastroenterol 2017;55:575-81.

5. Graham RP, Barr Fritcher EG, Pestova E, et al. Fibroblast growth factor receptor 2 translocations in intrahepatic cholangiocarcinoma. Hum Pathol 2014;45:1630-8.

6. Saha SK, Parachoniak CA, Ghanta KS, et al. Mutant IDH inhibits HNF-4 $\alpha$ to block hepatocyte differentiation and promote biliary cancer. Nature 2014;513:110-4.

7. Yang X, Wang W, Wang C, et al. Characterization of EGFR family gene aberrations in cholangiocarcinoma. Oncol Rep 2014;32:700-8.

8. Nathan H, Pawlik TM, Wolfgang CL, et al. Trends in survival after surgery for cholangiocarcinoma: a 30-year population-based SEER database analysis. J Gastrointest Surg 2007;11:1488-96; discussion 1496-7. 
9. Abou-Alfa GK, Sahai V, Hollebecque A, et al. Pemigatinib for previously treated, locally advanced or metastatic cholangiocarcinoma: a multicentre, open-label, phase 2 study. Lancet Oncol 2020;21:671-84.

10. Abou-Alfa GK, Macarulla T, Javle MM, et al. Ivosidenib in IDH1-mutant, chemotherapy-refractory cholangiocarcinoma (ClarIDHy): a multicentre, randomised, double-blind, placebo-controlled, phase 3 study. Lancet Oncol 2020;21:796-807.

11. Jakubowski CD, Azad NS. Immune checkpoint inhibitor therapy in biliary tract cancer (cholangiocarcinoma). Chin Clin Oncol 2020;9:2.

12. Valle J, Wasan H, Palmer DH, et al. Cisplatin plus gemcitabine versus gemcitabine for biliary tract cancer. $\mathrm{N}$ Engl J Med 2010;362:1273-81.

13. André T, Reyes-Vidal JM, Fartoux L, et al. Gemcitabine and oxaliplatin in advanced biliary tract carcinoma: a phase II study. Br J Cancer 2008;99:862-7.

14. Morizane C, Okusaka T, Mizusawa J, et al. Combination gemcitabine plus $\mathrm{S}-1$ versus gemcitabine plus cisplatin for advanced/recurrent biliary tract cancer: the FUGABT (JCOG1113) randomized phase III clinical trial. Ann Oncol 2019;30:1950-8.

15. Goldstein D, El-Maraghi RH, Hammel P, et al. nabPaclitaxel plus gemcitabine for metastatic pancreatic cancer: long-term survival from a phase III trial. J Natl Cancer Inst 2015;107:dju413.

16. Sahai V, Catalano PJ, Zalupski MM, et al. Nab-Paclitaxel and Gemcitabine as First-line Treatment of Advanced or Metastatic Cholangiocarcinoma: A Phase 2 Clinical Trial. JAMA Oncol 2018;4:1707-12.

17. Suenaga M, Yamada S, Fujii T, et al. S-1 plus nabpaclitaxel is a promising regimen for pancreatic cancer in a preclinical model. J Surg Oncol 2016;113:413-9.

18. Zhang W, Du C, Sun Y, et al. Nab-paclitaxel plus S-1 as first-line followed by S-1 maintenance for advanced pancreatic adenocarcinoma: a single-arm phase II trial. Cancer Chemother Pharmacol 2018;82:655-60.

19. Shi Y, Zhang S, Han Q, et al. Nab-paclitaxel plus S-1 in advanced pancreatic adenocarcinoma (NPSPAC): a single arm, single center, phase II trial. Oncotarget 2017;8:92401-10.

20. Malka D, Cervera P, Foulon S, et al. Gemcitabine and oxaliplatin with or without cetuximab in advanced biliarytract cancer (BINGO): a randomised, open-label, noncomparative phase 2 trial. Lancet Oncol 2014;15:819-28.

21. Sakai D, Kanai M, Kobayashi S, et al. Randomized phase III study of gemcitabine, cisplatin plus S-1 (GCS) versus gemcitabine, cisplatin (GC) for advanced biliary tract cancer (KHBO1401-MITSUBA). Ann Oncol 2018;29:viii205-viii270.

22. Shroff RT, Javle MM, Xiao L, et al. Gemcitabine, Cisplatin, and nab-Paclitaxel for the Treatment of Advanced Biliary Tract Cancers: A Phase 2 Clinical Trial. JAMA Oncol 2019;5:824-30.

23. Valle JW, Kelley RK, Furuse J, et al. KEYNOTE-966 trial in progress: pembrolizumab plus gemcitabine and cisplatin for advanced biliary tract cancer. Ann Oncol 2020;31:S270-1.

24. Ueno M, Ikeda M, Morizane C, et al. Nivolumab alone or in combination with cisplatin plus gemcitabine in Japanese patients with unresectable or recurrent biliary tract cancer: a non-randomised, multicentre, open-label, phase 1 study.

Lancet Gastroenterol Hepatol 2019;4:611-21.

25. Feng K, Liu Y, Zhao Y, et al. Efficacy and biomarker analysis of nivolumab plus gemcitabine and cisplatin in patients with unresectable or metastatic biliary tract cancers: results from a phase II study. J Immunother Cancer 2020;8:e000367.

26. Adams S, Diamond JR, Hamilton EP, et al. Phase Ib trial of atezolizumab in combination with nab-paclitaxel in patients with metastatic triple-negative breast cancer (mTNBC). J Clin Oncol 2016;34:abstr 1009.

27. Camidge R, Liu SV, Powderly J, Ready N, Hodi S, Gettinger SN, Giaccone G, Liu B, Wallin J, Funke R. ORAL02.07 Atezolizumab (MPDL3280A) Combined with Platinum-Based Chemotherapy in Non-Small Cell Lung Cancer (NSCLC): A Phase Ib Safety and Efficacy Update. J Thorac Oncol 2015;10;S176-7.

28. Valle JW, Wasan H, Johnson P, et al. Gemcitabine alone or in combination with cisplatin in patients with advanced or metastatic cholangiocarcinomas or other biliary tract tumours: a multicentre randomised phase II study - The UK ABC-01 Study. Br J Cancer 2009; 101:621-7.

29. Lamarca A, Ross P, Wasan HS, et al. Advanced Intrahepatic Cholangiocarcinoma: Post Hoc Analysis of the ABC-01, -02, and -03 Clinical Trials. J Natl Cancer Inst 2020;112:200-10.

Cite this article as: Zhang W, SunY, Jiang Z, Qu W, Gong C, Zhou A. Nab-paclitaxel plus tegafur gimeracil oteracil potassium capsule (S-1) as first-line treatment for advanced biliary tract adenocarcinoma: a phase 2 clinical trial. HepatoBiliary Surg Nutr 2023;12(1):37-44. doi: 10.21037/hbsn-21-172 